\title{
DUAS NOVAS ESPÉCIES DE SPOROBOLUS R. BR. (POACEAE - CHLORIDOIDEAE) DO BRASIL, RELACIONADAS COM S. CILIATUS PRESL
}

\author{
Hilda Maria Longhi-Wagner ${ }^{1}$ \\ Sonja de Castro Boechat ${ }^{2}$
}

Recebido em 11-11-92. Aceito em 01-12-93.

RESUMO: (Duas novas espécies de Sporobolus R. Br. [Poaceae - Chloridoideae] do Brasil, relacionadas com S. ciliatus Presl). Duas novas espécies de Sporobolus $\mathrm{R}$. Br. são descritas para o Brasil, $S$. metallicolus e $S$. multiramosus, a primeira distribuída desde o Brasil centro-oeste até o Estado do paraná, e a segunda só conhecida até o momento, do norte do Brasil (Pará). Devido ao seu pequeno porte e à inflorescência linear e contraída, ambas espécies são próximas de $S$. ciliatus Presl. Esta relação é discutida no presente trabalho.

Palavras-chave: Poaceae, Sporobolus, espécies novas, Brasil

ABSTRACT: (Two new species of Sporobolus R. Br. [Poaceae - Chloridoideae] from Brazil, related to S. ciliatus Presl). Two new species of Sporobolus R. Br. are described for Brazil, S. metallicolus and S. multiramosus, the first one distributed from CentralWestern Brazil to paraná State, the second only known so far for Northern Brazil (Pará State). Both species are closely related to $S$. ciliatus Presl due to their small size and their contracted inflorescence. This relationship is discussed in the present work.

Key-words: Poaceae, Sporobolus, new species, Brazil.

\section{Introdução}

O gênero Sporobolus $\mathrm{R}$. Br. inclui cerca de 100 espécies distribuídas nas regiões tropicais e temperadas de ambos os hemisférios. No Brasil ocorrem 28 espécies, das quais cinco citadas por Smith et al. (1981) para Santa Catarina, cinco citadas por Renvoize (1984) para a Bahia, seis delas tratadas com mais detalhe por Longhi-Wagner (1986,

1 - Departamento de Botânica UFRGS - Av. Paulo Gama, s.n. - 90049-060 - Porto Alegre - RS Brasil. Pesquisadora do $\mathrm{CNPq}$.

2 - Departamento de Botânica UFRGS. Trabalho subvencionado pelo CNPq e FAPERGS.. 
1990) para a Cadeia do Espinhaço e sete por Boechat \& Valls (1991) para o Rio Grande do Sul.

Os dois taxa aqui descritos, juntamente com S. ciliatus, formam um grupo típico de espécies, que se distinguem das demais ocorrentes no Brasil pelo pequeno porte aliado ao tipo de inflorescência. linear e contraída. Enquanto $S$. ciliatus tem uma distribuição restrita ao Brasil, e geralmente são encontradas sobre solos ricos em minério de ferro.

\section{Material e métodos}

Os dados apresentados neste trabalho são baseados em revisão de literatura e de herbários, bem como na análise da variabilidade das populações no campo, no caso de $S$. metallicolus.

Dos 32 herbários do Brasil e exterior examinados, foi encontrado material das duas novas espécies nos listados a seguir, citados pela sua sigla internacional: BHMH, ESAL, HB, IAN, IBGE, ICN, K, M, BM, MG, MO, NY, PEL, R, RB, SP, SPF, UB, US.

As medidas que aparecem entre parênteses nas descrições correspondem a valores extremos raramente encontrados no material.

\section{Resultados e discussão}

Sporobolus metallicolus Longhi-Wagner \& Boechat, sp. nov.

Figura 1: A-F

Perennis, caespitosa, $32 \mathrm{~cm}$ alta. Innovationes intravaginales. Culmi $2-3$ (4) nodi, ad basim ramosi. Vaginae foliorum glabrae, internodiis breviores. laminae $7-12 \mathrm{~cm}$ longi, 1,5-2 mm lati, marginibus ciliatis vel glabris. Ligula ciliata, $0,3-0,4 \mathrm{~mm}$ longa.

Panicula linearis, $8 \mathrm{~cm}$ longa, rami alterni, basales $0,8 \mathrm{~cm}$ longi, apicales $0,5-0,7 \mathrm{~cm}$ longi, inferne nudi. Spiculae 2,3-2,4 mm longae, 1-1,1 mm latae, pedicelli glandulosi vel non glandulosi. Glumae inferiores $1 \mathrm{~mm}$ longae, $0,15 \mathrm{~mm}$ latae, enerveae. Glumae superiores 2-2,2 $\mathrm{mm}$ longae, $0,4 \mathrm{~mm}$ latae, 1 -nervatae. Lemmata $2 \mathrm{~mm}$ longa, $0,3 \mathrm{~mm}$ lata. Paleae $1,8 \mathrm{~mm}$ longae, $0,7 \mathrm{~mm}$ latae. Stamina 3 , antherae $0,8 \mathrm{~mm}$ longae. Fructi $1-1,2 \mathrm{~mm}$ longae, $0,6 \mathrm{~mm}$ lati, non sulcati, pericarpium sementi adhaerens, non alatus.

Holótipo: Brasil - Minas gerais: ca. $10 \mathrm{~km} \mathrm{~W}$ de barão de Cocais, Serra do Caraça, 22.I.1971, H.S. Irwin et al. 28813, (MBM). Isótipos: UB, R, US.

Perenes, 15-40 cm alt., cespitosas, colmos 2-3.(4) nós. Inovações intravaginais. bainhas foliares glabras, menores do que os estrenós. Lâminas convolutas, lineares $(2,5)$ 4-11 cm compr., 1,5-3 $(3,2) \mathrm{mm}$ larg., geralmente com relação comprimento $\mathrm{x}$ largura maior do que 20 (20-45), escabras, raramente com pêlos esparsos na face ventral, com ou sem cílios longos (2,5-3 mm) e pectinados nos bordos. Lígula ciliada, 0,3-0,5 $\mathrm{mm}$ compr.; bordos da região ligular glabros. 
Panícula 5,5-8 cm compr., linear, estreita, ramos alternos, desprovidos de espiguetas na base, os inferiores $0,8-1,5 \mathrm{~cm}$ compr., os superiores $(0,5)$ ),7-1,1 cm compr., Inflorescências axilares ausentes. Eixo da inflorescência e pedicelos glandulosos. Espiguetas 2-2,6 mm compr., 0,8-1,3 mm larg., cor estramínea. Gluma inferior estreito acuminada, enérvea $(0,8) 1-1,5 \mathrm{~mm}$ compr., $0,15-0,2 \mathrm{~mm}$ larg., alcançando no máximo a metade do antécio; gluma superior aguda, 1(2)-nervada, 2-2,6 mm compr., 0,4-0,6 mm larg., de comprimento igual ou sobrepassando $o$ antécio. lema (1,8) 2-2,3 mm compr., 0,3-0,4 mm larg., uninervado, agudo. Pálea 1,5-2,2 mm compr., 0,8-1,0 mm larg., 2-nervada, bordos hialinos, as duas metades separando-se por pressão da cariópse madura. Estames 3, anteras 0,8-1,0 mm compr. Fruto 1-1,3 mm compr., 0,5-0,7 mm larg., oblongo, sem sulco, geralmente desprendendo-se inteiro da inflorescência. Pericarpo envolvendo apertadamente a semente, raramente formando uma ala estreita.

Sporobolus metallicolus foi citada por Longhi-Wagner $(1986,1990)$ como S. aff. piliferus, chamando a atenção para a sua semelhança com S. piliferus (Trin.) Kunth, espécie descrita por Trinius (1828) como tendo uma distribuição disjunta entre o Nepal, com uma forma de menor porte, e o Brasil, com uma forma de maior porte. Trinius (1828) não citou o material examinado, porém ilustrou um exemplar de cada região. Kunth (1833) descreveu estas duas formas como variedades de S. piliferus, variedades minutus, e major. O espécime brasileiro ilustrado por Trinius (1828) representa uma planta perene, com características semelhantes ao material aqui descrito. Por outro lado, o material asiático ilustrado, corresponde a uma planta anual, com inflorescência e espiguetas semelhantes ao material brasileiro. Exemplares de S. piliferus analisados no herbário $\mathrm{K}$, todos procedentes de montanhas rochosas da África, em altitudes acima de $1000 \mathrm{~m}$ s.n.m., confirmam o caráter anual da espécie, a qual apresenta lâminas foliares um pouco mais largas do que $S$. metallicolus, com pêlos longos esparsos nas duas faces, gluma inferior um pouco maior, com 1,5-2 mm de comprimento, e cariópse com estrias longitudinais bem marcadas. Portanto, S. metallicolus, espécie perene, parece corresponder à forma brasileira de S. piliferus descrita por Trinius (1828), e é considerada neste trabalho uma espécie independente, especialmente devido às diferenças no ciclo de vida $\mathrm{e}$ na distribuição geográfica.

Vários exemplares de $S$. metaliicolus, nos herbários revisados, estavam identificados com S. rupestris Kunth. Embora não tenha sido possível localizar o exemplar-tipo desta última espécie, a análise de sua descrição original, que a indica como espécie anual, e da ilustração do hábito do holótipo, com detalhes da espigueta e do fruto, apresentada por Kunth (1830), permitiu verificar que corresponde a S. ciliatus, sinonímia já proposta anteriormente por Longhi-Wagner (1990).

Sporobolus ciliatus (S. rupestris) difere de S. metallicolus por ser anual, pelas lâminas foliares mais curtas e largas, com índice comprimento x largura igual a 6-15 (22), geralmente pilosas nas duas faces, cariópse suborbicular, pouco comprimida lateralmente, com pericarpo envolvendo frouxamente a semente, formando uma ala em torno da mesma. Em S. metallicolus, o índice comprimento x largura das lâminas foliares é geralmente 
maior do que 20, as lâminas apresentam apenas cílios marginais, e a cariópse é oblonga e comprimida lateralmente, com o pericarpo envolvendo apertadamente a semente, em geral sem formar ala ou mais raramente formando uma ala estreita.

Utilizando os caracteres referidos acima foi elaborado um diagrama de dispersão (Figura 2) para salientar as diferenças entre as duas espécies. neste diagrama foram incluídos dados de 26 espécimes, os quais formaram dois grupos definidos, correspondentes às duas entidades taxonômicas.

Sporobolus mettalicolus é mais frequente nos campos rupestres da Cadeia do Espinhaço, em solos oriundos de quartzitos ou solos do tipo "canga", ricos em minério de ferro. Também foi coletada, embora com menor expressão, em campos de altitude do Estado do Paraná.

Parátipos: Brasil - Minas Gerais: Barbacena, 28.II.-3.III.1925, Chase 8648 (MO, RB, US); Belo Horizonte, 21.III.1925, Chase 8967 (MO, US); 13.I.1971, Irwin et al. 30223 (MO, UB); Betim-Brumadinho, 5.III.1940, Oliveira s/n (IAN 15001); Brumadinho, 5.III.1940, Mello-Barreto 10977 (BHMH); Catas Altas, 24.II.1944, Black \& Pereira 2506B (US), Catas Altas-Santa Bárbara, 5.II.1943, Mag 2850 (IAN); Itabira, 21.X.1888, Glaziou 17887 (K, US); Itabirito, 11.II.1968, Irwin et al. 19857 (K, UB, US); Jaboticatubas. 11.II.1969, Eiten \& Eiten 11015 (SP); 16.IV.1972, CFSC 1847, Joly et al. (SP); Lavras, 26.II.1944, Black 2233B (ESAL); 24.II.1942, Black 2506 (ESAL); 26.II.1944, Black 2561 (ESAL); Lavras Novas, III.1893, Damázio s/n (RB 203255); Lima Duarte, 3.XII.1987, Andrade 917 (IBGE); Ouro Preto, 13-15.II.1978, Burman 127 (SP); 8.IV.1925, Chase 9387 (US); II.1894, Damázio 2121 (R, RB); s/ data, Damázio s/n (RB 183395, 189400), 30.I.1971, Irwin et al. 29452 (R, UB, US); 20.I.1951, Macedo 3005 (MO, SP, US); 23.I.1972, Macedo 5224 (HB); 27.I.1972, Macedo 5240 (HB); Santana do Riacho, 27.I.1986, CFCR 9578, Longhi-Wagner et al. (ICN, SPF); I. 1951., Pires \& Black 2926 (US); I. 1951, Pires \& Black 3035 (IAN); Serra do Cipó, 25.XI.1979, Burman 517 (SP), São Tomé das Letras, 11.I.1969, Monteiro 6699 (K); São João del Rei, Serra do Lenheiro, 10.IV.1988, Glaziou 17439 (K); s/local, Serra do Espinhaço, 11.II.1968, Irwin et al. 19857 (MO); 22.I.1971, Irwin et al. 28813 (HB); 30.I.1971, Irwin et al. 29452 (HB). Paraná: Guarapava, 21.III.1946, Swallen 8836 (PEL). S/local, Serra da Gramma, 19-25.IV.1925, Chase 9558 (MO); s/local, s/data, Glaziou 15625 (US); s/local, s/data, Langsdorff s/n (US 998707).

Material adicional examinado: Sporobolus piliferus (Trin.) Kunth: África, Zâmbia: Malawi, Chitipa Dist., Misuku Hills, 8.VII.1973, J. Pawek 7105 (K); Mzimba Dist., Mzuzu, Marymount, 29.III.1974, J. Pawek 8252 (K); Mlange moutain, 6.IV.1970, R.K. Brummit 9688 (K). Rodésia: Inyanga Dist., IV.1969, W.B. Cleghorn 1995 (K); Juliasdale, 16.III.1975, Crook 2075 (K); Salisbury, 10.II.1974, Davidse \& B. Simon 6455 (K).

Sporobolus multiramosus Longhi-Wagner \& Boechat, sp. nov.

Figura 1: G-L 

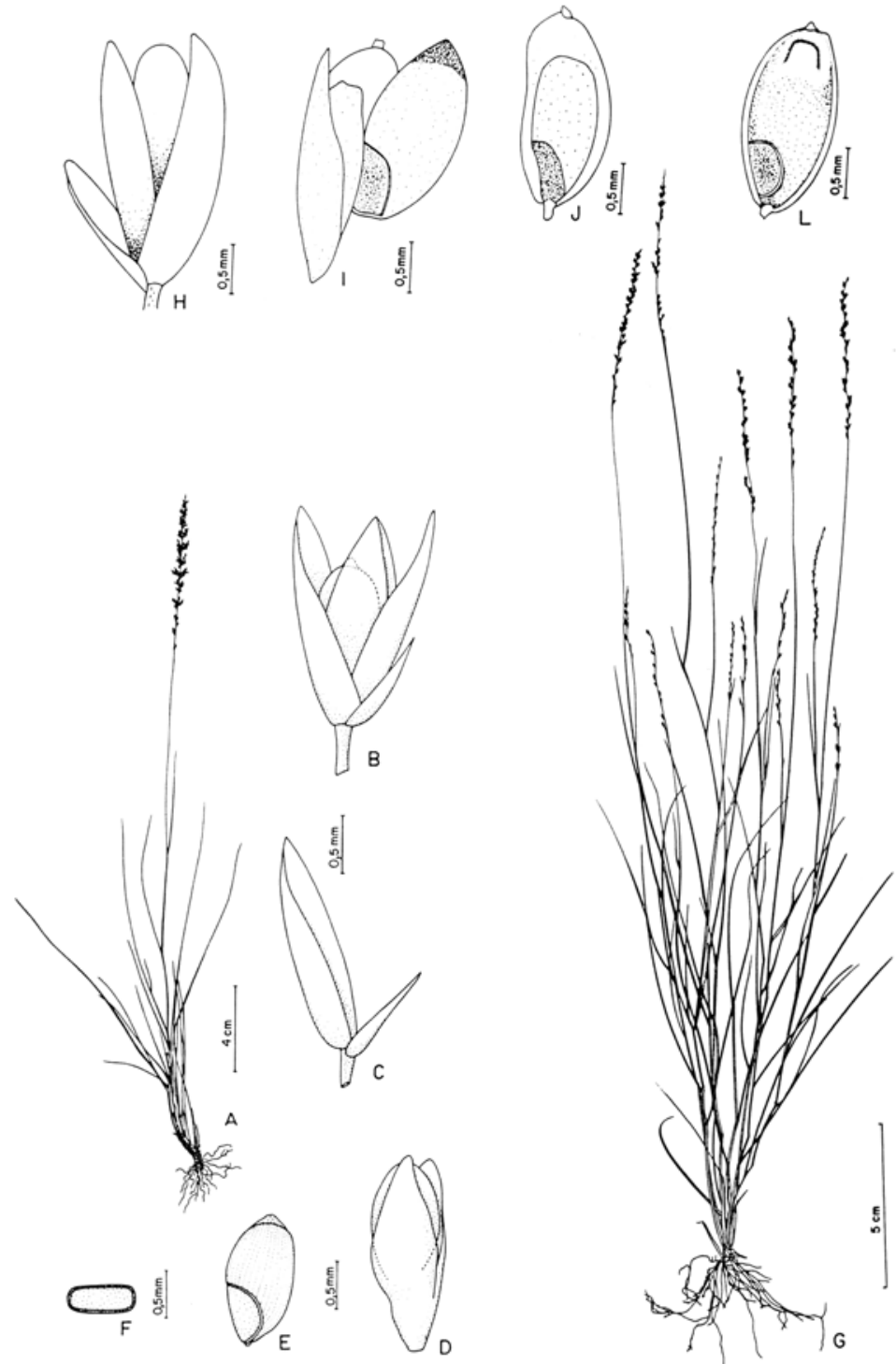

Figura 1 - Sporobolus metallicolus. A - hábito; B - espigueta com fruto; C - glumas; D - pálea partida longitudinalmente por pressão no fruto; $\mathrm{E}$ - fruto, vista lateral; F - fruto; corte longitudinal (Irwin et al. 28813 - MBM, holótipo). Sporobolus multiramosus - G - hábito; H - espigueta; I lema, pálea partida longitudinalmente por pressão do fruto, semente liberada do pericarpo; $\mathrm{J}$ - fruto com o pericarpo frouxamente aderido à semente; $\mathrm{L}$ - fruto, vista lateral $(\mathrm{G}, \mathrm{H}, \mathrm{L}$, - Rosa \& Silva 4680 -SP, holótipo; I, J - Cavalcante 2125, US). 
Annua, $38 \mathrm{~cm}$ alta, caespitosa. Culmi graciles, 7-nodi, praesertim ramosi ad nodos medianos. Vaginae foliorum glabrae, internodi breviores. Laminae 3-7 cm longae, 1-1,5 $\mathrm{mm}$ latae, glabrae. Ligula ciliata, $0,4 \mathrm{~mm}$ longa.

Panicula linearis, $3-5,5 \mathrm{~cm}$ longa, $0,2-0,3 \mathrm{~cm}$ lata, pedunculi $0,4-3 \mathrm{~cm}$ longi; rami alterni, basales $0,4-0,5 \mathrm{~cm}$ longi, apicales $0,1 \mathrm{~cm}$ longi, inferne nudi. Paniculae axilares frequentes. Spiculae 2,3-2,4 mm longae, 0,8-1 mm latae. Glumae inferiores 1,5-1,7 mm longae, 0,1-0,2 mm latae, enerviae. Glumae superiores 2-2,6 mm longae, 0,4 mm latae, 1-3 nervatae. Lemmata 2-2,2 mm longa, 0,4 mm lata. Paleae 1,8-2,2 mm longae, 0,2-0,5 $\mathrm{mm}$ latae. Staminae 2-3, antherae $1 \mathrm{~mm}$ longae. Fructi 1,7-2 $\mathrm{mm}$ longi, 0,7-0,9 mm lati, non sulcati, pericarpum seminis leviter adhaerens, alatus vel non alatus.

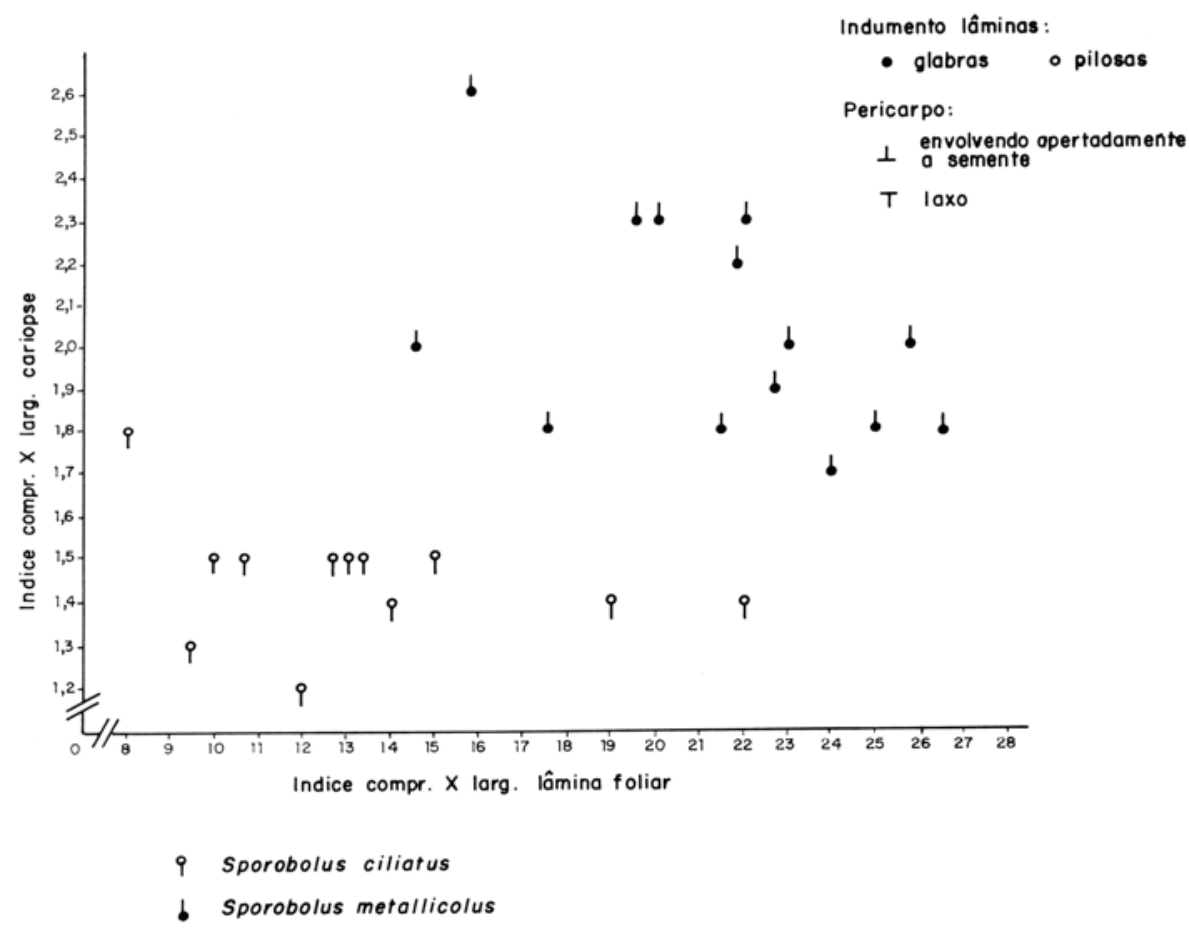

Figura 2 - Diagrama de dispersão com análise de S. ciliatus ( ( ) e S. metallicolus ( b). Número de espécimes incluídos: 26 . 
Holótipo: Brasil - Pará: Marabá, 19.IV.1985, Rosa \& Silva 4680 (SP).

Anuais, $18-50 \mathrm{~cm}$ alt., ceaspitosas, delicadas, colmos frequentemente geniculados, muito delgados, 0,5 mm diâmetro, 6-7 nós, tipicamente ramificados nos nós superiores, especialmente nos medianos. Inovações intravaginais. Bainhas foliares glabras, menores que os entrenós. Lâminas foliares lineares, planas, conduplicadas, involutas, ou convolutas, 2-13 cm compr., 1-1,8 mm larg., ciliadas nos bordos ou glabras. Lígula ciliada, 0,3-0,4 $\mathrm{mm}$ compr., bordos da região ligular com pêlos de 1-1,2 mm compr., subdensos.

Panícula 3-7 cm compr., 0,2-0,3 cm larg., linear, estreita, ramos alternos, filiformes, os inferiores $0,4-1,3 \mathrm{~cm}$ compr., os superiores $0,1-0,2 \mathrm{~cm}$ compr., desprovidos de espiguetas na base. Inflorescências axilares frequentes. Eixo da inflorescência e pedicelos glandulosos. Espiguetas 2-2,4 mm compr., 0,8-1 mm larg., cor estramínea. Glumas caducas, a inferior igual ou maior que a metade do comprimento da superior, enérvea, 1,1-1,7 mm compr., 0,1-0,2 mm larg.; gluma superior 1-3-nervada, 2-2,6 mm compr., $0,5 \mathrm{~mm}$ larg. Lema 2$2,2 \mathrm{~mm}$ compr., 0,3-0,5 mm larg., uninervado. Pálea 1,8-2,2 mm compr., 0,2-0,5 mm larg., 2-nervada, as duas metades separando-se por pressão da cariópse madura. Estames 3 , anteras $0,5-0,8 \mathrm{~mm}$ compr. Fruto $1,7-2 \mathrm{~mm}$ compr., $0,7-1 \mathrm{~mm}$ larg.,oblongo, castanho, sem sulco. Pericarpo envolvendo frouxamente a semente, às vezes esbranquiçado quando afastado da mesma e facilmente removível, formando ou não uma ala em torno da mesma, às vezes inflado no ápice do fruto.

Sporobolus multiramosus distingue-se de S. ciliatus e de S. metallicolus pelo maior número de nós nos colmos floríferos, com ramificação típica superiormente, em especial nos nós medianos, por apresentar inflorescências axilares frequentes e pelas lâminas foliares mais estreitas. Além disto, difere de S. metallicolus por ser uma espécie anual, como S. ciliatus, a qual estretanto apresenta lâminas foliares mais largas, com $(1,2)$ 2,0-4,5 mm compr., pilosas nas duas faces. Sporobolus multiramosus se aproxima de S. temomairemensis Judziewicz \& Peterson, descrita do Amapá, no norte do Brasil, pelo pequeno porte e colmos delicados, com lâminas foliares muito estreitas. Sporobolus temomairemensis, porém, apresenta, segundo Judziewicz \& Peterson (1989), panícula aberta, sem inflorescências axilares, ambas as glumas menores do que 0 antécio, espiguetas menores, com (0,9) 1,0-1,3 mm compr., e fruto 0,7-1 mm compr.

A nova espécie tem sua ocorrência, até o momento, só conhecida para o Estado do Pará, no norte do Brasil, ocorrendo em vegetação rupestre, em altitudes de 650 m s.n.m., sobre solos ricos em minério de ferro.

Parátipos: Brasil - Pará: Marabá, 19.V.1987, Bastos \& Rosa 474 (SP); 22.V.1969, Cavalcante 2125 (US); 18.IV.1970, Cavalcante \& Silva 2626 (US); 14.V.1982, Secco et al. 151 (MO); 17.V.1982, Secco et al. 218 (MO, NY); 17.III.1985, Secco et al. 478 (MG); s/local, Serra dos Carajás, 13.V.1982, Sperling et al. 5650 (MO).

\section{Agradecimentos}

Ao Dr. Tarciso Filgueiras pela correção das descrições latinas. 


\section{Referências bibliográficas}

Boechat, S.C. \& J.F.M. Valls. 1991. As espécies do gênero Sporobolus (L.) R. Br. (Gramineae, Chloridoideae) no Rio Grande do Sul. Iheringia, ser. Bot. 41: 9-45.

Judziewicz, E.J. \& P.M. Peterson. 1989. Sporobolus temomairemensis (Poaceae: Eragrostideae): a new species from Northern South America. Systematic Botany 14: 525-528.

Kunth, C.S. 1830. Revision des Graminées, Paris.

Kunth, C.S. 1833. Enumeratio Plantarum: Agrostographia synoptica. J.G. Cottae, Stutgardiae et Tubingae. v. 1.

Longhi-Wagner, H.M. 1986. A subfamilia Chloridoideae (Gramineae) na Cadeia do Espinhço, Brasil. 627p. Tese de doutorado, Universidade de São Paulo, São Paulo (não publicada).

Longhi-Wagner, H.M. 1990. Flora da Serra do Cipó, Minas Gerais: Gramineae I -

Chloridoideae. Bol. Botânica Univ. São Paulo 12: 15-42.

Renvoize, S. 1984. The Grasses of Bahia. Royal Botanic Gardens, Kew.

Smith, L.B.; D.C. Wasshausen \& R.M. Klein. 1981. Gramíneas. Flora Ilustrada Catarinense. (Gram.)

Trinius, C.B. 1828. Species Graminum. Iconibus et descriptionibus. Impr. Acad. Imp. Scient. , Petropoli. v. 2. Reprint 1970, J. Cramer, New York. 